\title{
Universality of electron mobility in LaAIO3/SrTiO3 and bulk SrTiO3
}

Trier, Felix; Reich, K. V.; Christensen, Dennis Valbjørn; Zhang, Yu; Tuller, Harry L.; Chen, Yunzhong; Shklovskii, B. I.; Pryds, Nini

Published in:

Applied Physics Letters

Link to article, DOI:

$10.1063 / 1.5001316$

Publication date:

2017

Document Version

Publisher's PDF, also known as Version of record

Link back to DTU Orbit

Citation (APA):

Trier, F., Reich, K. V., Christensen, D. V., Zhang, Y., Tuller, H. L., Chen, Y., Shklovskii, B. I., \& Pryds, N. (2017). Universality of electron mobility in $\mathrm{LaAlO}_{3} / \mathrm{SrTiO}_{3}$ and bulk $\mathrm{SrTiO}_{3}$. Applied Physics Letters, 111(9), [092106]. https://doi.org/10.1063/1.5001316

\section{General rights}

Copyright and moral rights for the publications made accessible in the public portal are retained by the authors and/or other copyright owners and it is a condition of accessing publications that users recognise and abide by the legal requirements associated with these rights.

- Users may download and print one copy of any publication from the public portal for the purpose of private study or research.

- You may not further distribute the material or use it for any profit-making activity or commercial gain

- You may freely distribute the URL identifying the publication in the public portal 


\section{Universality of electron mobility in $\mathrm{LaAlO}_{3} / \mathrm{SrTiO}_{3}$ and bulk $\mathrm{SrTiO}_{3}$}

Felix Trier, K. V. Reich, Dennis Valbjørn Christensen, Yu Zhang, Harry L. Tuller, Yunzhong Chen, B. I. Shklovskii, and Nini Pryds

Citation: Appl. Phys. Lett. 111, 092106 (2017);

View online: https://doi.org/10.1063/1.5001316

View Table of Contents: http://aip.scitation.org/toc/apl/111/9

Published by the American Institute of Physics

\section{Articles you may be interested in}

Enhancing the barrier height in oxide Schottky junctions using interface dipoles

Applied Physics Letters 111, 091602 (2017); 10.1063/1.4991691

Room temperature 2D electron gas at the (001)- $\mathrm{SrTiO}_{3}$ surface

Applied Physics Letters 111, 181601 (2017); 10.1063/1.5001222

Suppressed carrier density for the patterned high mobility two-dimensional electron gas at $\gamma_{-}-\mathrm{Al}_{2} \mathrm{O}_{3} / \mathrm{SrTiO}_{3}$ heterointerfaces

Applied Physics Letters 111, 021602 (2017); 10.1063/1.4993165

Near-infrared luminescence in perovskite $\mathrm{BaSnO}_{3}$ epitaxial films

Applied Physics Letters 111, 091903 (2017); 10.1063/1.4997344

Patterning the two dimensional electron gas at the $\mathrm{LaAlO}_{3} / \mathrm{SrTiO}_{3}$ interface by structured Al capping Applied Physics Letters 110, 141603 (2017); 10.1063/1.4979784

Direct optical observation of spin accumulation at nonmagnetic metal/oxide interface Applied Physics Letters 111, 092402 (2017); 10.1063/1.4990113

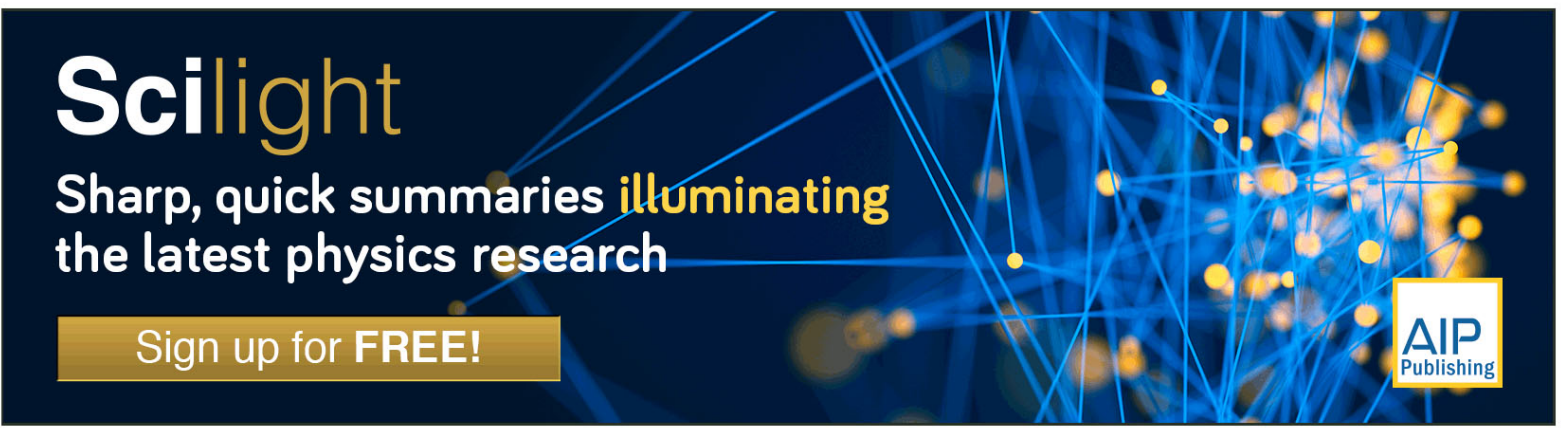




\title{
Universality of electron mobility in $\mathrm{LaAlO}_{3} / \mathrm{SrTiO}_{3}$ and bulk $\mathrm{SrTiO}_{3}$
}

\author{
Felix Trier, ${ }^{1, a)}$ K. V. Reich, ${ }^{2,3}$ Dennis Valbjørn Christensen, ${ }^{1}$ Yu Zhang, ${ }^{1}$ Harry L. Tuller, ${ }^{4}$ \\ Yunzhong Chen, ${ }^{1}$ B. I. Shklovskii, ${ }^{2}$ and Nini Pryds ${ }^{1, b)}$ \\ ${ }^{1}$ Department of Energy Conversion and Storage, Technical University of Denmark, Ris $\phi$ Campus, \\ 4000 Roskilde, Denmark \\ ${ }^{2}$ Fine Theoretical Physics Institute, University of Minnesota, Minneapolis, Minnesota 55455, USA \\ ${ }^{3}$ Ioffe Institute, St Petersburg, 194021, Russia \\ ${ }^{4}$ Department of Materials Science and Engineering, Massachusetts Institute of Technology, Cambridge, \\ Massachusetts 02139, USA
}

(Received 12 May 2017; accepted 23 August 2017; published online 31 August 2017)

\begin{abstract}
Metallic $\mathrm{LaAlO}_{3} / \mathrm{SrTiO}_{3}$ (LAO/STO) interfaces attract enormous attention, but the relationship between the electron mobility and the sheet electron density, $n_{s}$, is poorly understood. Here, we derive a simple expression for the three-dimensional electron density near the interface, $n_{3 D}$, as a function of $n_{s}$ and find that the mobility for LAO/STO-based interfaces depends on $n_{3 D}$ in the same way as it does for bulk doped STO. It is known that undoped bulk STO is strongly compensated with $N \simeq 5 \times 10^{18} \mathrm{~cm}^{-3}$ background donors and acceptors. In intentionally doped bulk STO with a concentration of electrons $n_{3 D}<N$, background impurities determine the electron scattering. Thus, when $n_{3 D}<N$, it is natural to see in LAO/STO the same mobility as in the bulk. On the other hand, in the bulk samples with $n_{3 D}>N$, the mobility collapses because scattering happens on $n_{3 D}$ intentionally introduced donors. For LAO/STO, the polar catastrophe which provides electrons is not supposed to provide an equal number of random donors and thus the mobility should be larger. The fact that the mobility is still the same implies that for the LAO/STO, the polar catastrophe model should be revisited. Published by AIP Publishing. [http://dx.doi.org/10.1063/1.5001316]
\end{abstract}

Recently, much attention has been directed at metallic $\mathrm{LaAlO}_{3} / \mathrm{SrTiO}_{3}$ (LAO/STO) interfaces. ${ }^{1-5}$ Such interfaces have been shown to exhibit a plethora of physical phenomena. These include gate-tunable superconductivity, magnetism, metal-insulator transitions, and quantized Hall resistance. ${ }^{6-13}$ Motivated by interest in quantum phenomena and potential applications, multiple studies have specifically sought to improve the low-temperature electron mobility in LAO/STO interfaces. ${ }^{13-21}$ In spite of more than a decade of research, the dominant scattering mechanism in LAO/STO interfaces, nonetheless, remains elusive.

The canonical $\mathrm{LaAlO}_{3} / \mathrm{SrTiO}_{3}$ interface (LAO/STO) has previously been shown to exhibit large variations of the lowtemperature electron mobility $\mu$ and sheet electron density $n_{s}$, which depend strongly on interface growth conditions. ${ }^{19,22}$ Remarkably in these previous studies, the dependence of $\mu\left(n_{s}\right)$ seems to follow a universal behaviour, ${ }^{19,22}$ almost regardless of the type of LAO/STO interface and particular growth conditions (see blue markers in Fig. 1).

The aim of this paper is to relate $\mu\left(n_{s}\right)$ data for LAO/STObased interfaces from the literature ${ }^{13,16-19,21-23}$ and from newly prepared by us LAO/STO-based samples with the available electron mobility data for bulk doped STO over a range of three-dimensional electron densities from the literature ${ }^{24-29}$ (see red markers in Fig. 1). To this end, we note that at low temperature, the electrons are distributed in a layer of width, $d \simeq 5-100 \mathrm{~nm}^{30-34}$ near the LAO/STO interface. Due to the relatively large width of this electron layer, we essentially deal

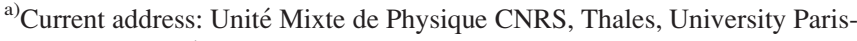
Sud, Université Paris-Saclay, 91767 Palaiseau, France. Electronic mail: felix.trier@cnrs-thales.fr

b)Email: nipr@dtu.dk
}

with a three-dimensional system. ${ }^{35,36}$ Such an electronic system can be described by the recent theory ${ }^{37-39}$ of accumulation layers in STO based on a combination of the Landau-Ginzburg description of the dielectric response of the STO lattice and the Thomas-Fermi approximation for the degenerate electron gas. Using this theory, we show below that the three-dimensional electron density of the electronic system near the LAO/STO interface, $n_{3 D}$, depends on the measured two-dimensional sheet electron density, $n_{s}$, in the following way:

$$
n_{3 D}\left(n_{s}\right)=\frac{C\left(n_{s} a^{2}\right)^{6 / 5}}{\left(a_{B} a^{4}\right)^{3 / 5}}\left[1+\frac{A \kappa}{8 \pi}\left(n_{s} a^{2}\right)^{2}\right]^{3 / 5} .
$$

Here, $C \simeq\left(5^{3} 2^{3} 3^{-2} \pi^{-1}\right)^{1 / 5} \simeq 2, a \simeq 3.9 \AA$ is the lattice constant of STO, $a_{B}=\hbar^{2} 4 \pi \varepsilon_{0} \kappa / m^{\star} e^{2} \simeq 7000 \AA$ is the effective Bohr radius, $\kappa \simeq 20000$ is the dielectric constant of STO at low temperatures, the coefficient $A \simeq 0.9$ describes the non-linear dielectric response of STO, and $m^{\star} \simeq 1.6 m_{e}$ is the effective electron mass in STO, ${ }^{12,40,41}$ with $m_{e}$ being the free electron mass. For example, a three-dimensional density of $n_{3 D}=5 \times 10^{18} \mathrm{~cm}^{-3}$ corresponds to a two-dimensional sheet density of $n_{s}=1.5 \times 10^{13} \mathrm{~cm}^{-2}$.

In Fig. 1, by using Eq. (1), we compare the low temperature electron mobility for LAO/STO-based interfaces with mobility for bulk doped STO having the same electron density $n_{3 D}$. We see that the values of these mobilities and the concentration dependencies for LAO/STO-based interfaces and bulk STO are similar. This suggests that the electron scattering mechanisms in LAO/STO-based interfaces are the same as those in bulk STO.

In the following, we briefly review known mechanisms for electron scattering in bulk conducting STO at low 


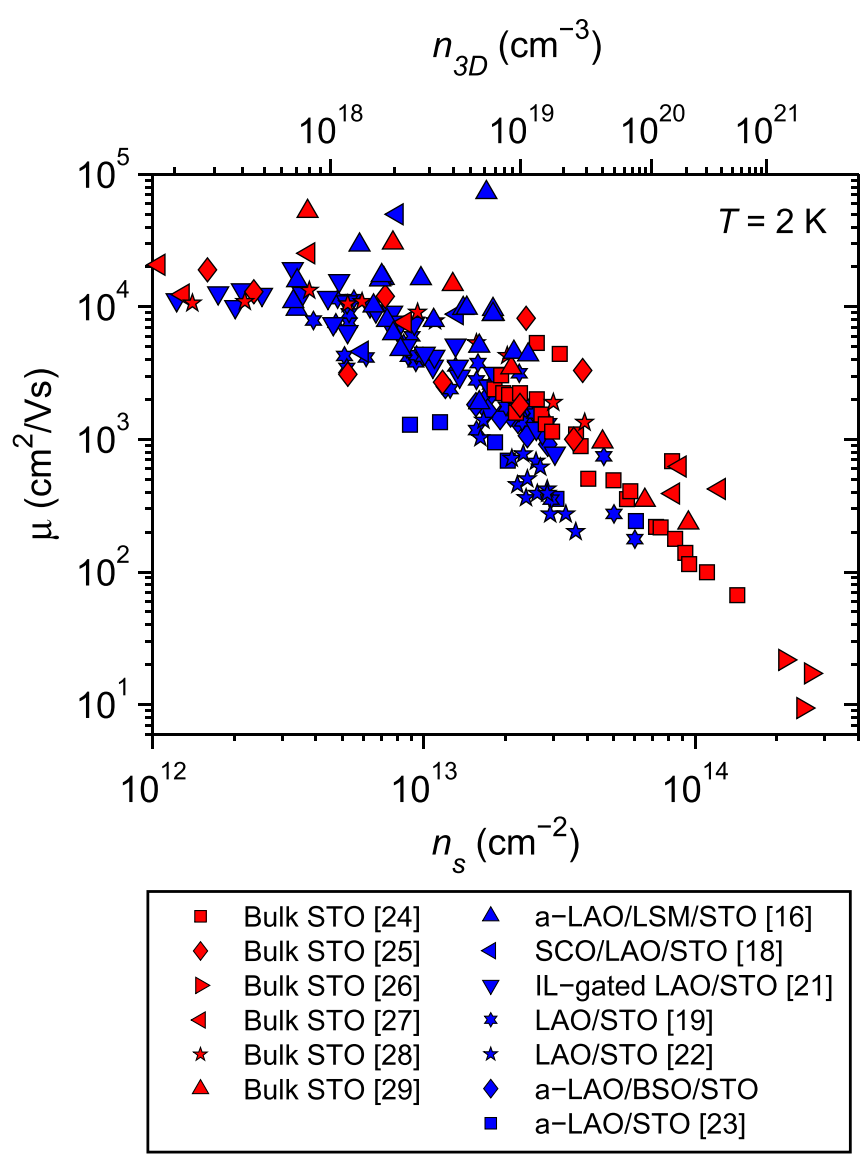

FIG. 1. The dependence of the low-temperature electron mobility, $\mu$, on the sheet carrier density, $n_{s}$, (lower scale) and bulk carrier density, $n_{3 D}$, (upper scale). The data for bulk STO (red markers) and LAO/STO-based interfaces (blue markers) are close to each other. The data encompass the following STO-based systems: bulk STO; ${ }^{24-29}$ amorphous- $\mathrm{LaAlO}_{3} / \mathrm{LaSrMnO}_{3} / \mathrm{SrTiO}_{3}$ (a-LAO/LSM/STO), Ref. 16 and this work; $\mathrm{SrCuO}_{2} / \mathrm{LaAlO}_{3} / \mathrm{SrTiO}_{3}(\mathrm{SCO} /$ $\mathrm{LAO} / \mathrm{STO}$ ); ${ }^{18}$ ion liquid-gated $\mathrm{LaAlO}_{3} / \mathrm{SrTiO}_{3}$ (IL-gated LAO/STO); ${ }^{21}$ $\mathrm{LaAlO}_{3} / \mathrm{SrTiO}_{3}$ (LAO/STO); ${ }^{19,22}$ amorphous-LaAlO $/ \mathrm{BaSnMnO}_{3} / \mathrm{SrTiO}_{3}$ (a-LAO/ BSO/STO), this work; amorphous- $\mathrm{LaAlO}_{3} / \mathrm{SrTiO}_{3}$ (a-LAO/STO), Ref. 23 and this work.

temperatures. ${ }^{25,29}$ Let us first dwell on the interpretation of the electron mobility in nominally insulating bulk STO. It is well established that bulk STO samples usually are strongly compensated, ${ }^{25,27,42}$ i.e., have nearly equal donor and acceptor concentrations. In bulk STO, the possible background donors include oxygen vacancies, while common acceptors include strontium vacancies, aluminium, or iron. ${ }^{42-44}$ Due to a transition of electrons from donors to acceptors, the total concentration of charged background impurities can be as large as $N \sim 5 \times 10^{18} \mathrm{~cm}^{-3}$. ${ }^{42}$ This means that in both bulk STO and LAO/STO-based interfaces with $n_{3 D} \ll N$ (or $n_{s}$ $\ll 1.5 \times 10^{13} \mathrm{~cm}^{-3}$ ), the electron mobility is limited by electrons scattering on background charged impurities. For bulk STO having $n_{3 D} \gg N$ (or $n_{s} \gg 1.5 \times 10^{13} \mathrm{~cm}^{-3}$ ), the lowtemperature electron mobility is instead limited by scattering on intentionally added ionized donors. On the one hand, these ionized donors provide free charge carriers to the electronic system; on the other hand, they act as scattering centres. If we assume that in LAO/STO-based interfaces with $n_{3 D}>N$, electrons are provided by a conventional polar catastrophe, which does not bring positive random donors together with them; mobility of such samples should be much larger than that of the bulk STO with the same 3D concentration. Thus, the overall universality of the electron mobility in Fig. 1 allows us to conclude that electrons in LAO/STO-based interfaces with $n_{3 D}>N$ are provided not by the polarization catastrophe but by the equal number of donors which scatter electrons. This observation questions the polarization catastrophe model and requires its further adjustment. For the case of amorphous-LAO/STO grown at room temperature, the role of ionized donors is undoubtedly played by oxygen vacancies in STO located near the interface. $^{23}$

Let us now dwell on our experimental details. The a-LAO/ STO, a-LAO/BSO/STO, and a-LAO/LSM/STO samples included in this study were all prepared on $\mathrm{TiO}_{2}$-terminated and (100)-oriented $\mathrm{SrTiO}_{3}$ (STO) substrates $\left(5 \times 5 \times 0.5 \mathrm{~mm}^{3}\right.$, miscut angle $<0.2^{\circ}$ ). For the a-LAO/BSO/STO and a-LAO/ LSM/STO samples, a single unit cell epitaxial $\mathrm{BaSnO}_{3}$ (BSO) or $\mathrm{La}_{7 / 8} \mathrm{Sr}_{1 / 8} \mathrm{MnO}_{3}$ (LSM) spacer layer, respectively, was deposited by pulsed laser deposition (PLD) in an oxygen atmosphere of $\sim 10^{-4}$ mbar at $600^{\circ} \mathrm{C}$. The film growth rate of LSM and BSO was determined from in-situ RHEED oscillations. Furthermore, the LSM and BSO were ablated from ceramic LSM and BSO targets with a target-substrate distance of $5.7 \mathrm{~cm}$, by a $\mathrm{KrF}$ laser $(\lambda=248 \mathrm{~nm})$ with a repetition rate of $1 \mathrm{~Hz}$ and a laser fluence of $1.5 \mathrm{~J} \mathrm{~cm}^{-2}$. The respective samples were then cooled under $\sim 10^{-4}$ mbar oxygen pressure at a rate of $15^{\circ} \mathrm{C} / \mathrm{min}$ to room temperature $\left(<25^{\circ} \mathrm{C}\right)$. Finally, $16 \mathrm{~nm}$ amorphous- $\mathrm{LaAlO}_{3}$ (a-LAO) was grown at an oxygen pressure of $\sim 10^{-6}$ mbar and room temperature on the respective samples to finalize the a-LAO/STO, a-LAO/BSO/STO, and a-LAO/ LSM/STO heterostructures. The a-LAO was ablated from a single crystalline LAO target, and otherwise identical PLD conditions were used as for BSO and LSM. All samples were mounted on ceramic chip-carriers and electrically connected in the van der Pauw geometry. The interface of all samples was contacted using ultrasonically wire-bonded aluminum wires. Sheet resistance and Hall resistance measurements between 2 and $300 \mathrm{~K}$ were performed using a standard DC technique $\left(I_{D C}=1-5 \mu \mathrm{A}\right)$ in a cryostat with magnetic fields up to $15 \mathrm{~T}$.

Note that all the data used in Fig. 1 are obtained for the LAO layers grown on the (100) oriented STO. Although the thickness of these LAO layers varies, the mobility has the same dependence on the concentration. We did not include LAO layers grown on (110) or (111) oriented STO, such as in Ref. 45. We found that even in such samples, the dependence of the mobility on the concentration is the same as in Fig. 1, with exception of the thickest sample. It shows very small mobility, possibly because of large surface roughness.

Below, we present the derivation of Eq. (1), which allowed LAO/STO versus bulk STO mobility comparison. The three-dimensional distribution of electrons near the STO interface, $n(x)$, was found in Ref. 37 in the two limiting cases of small and large two-dimensional electron densities $n_{s}$. For our purpose, we need the three-dimensional electron density near the interface $n_{3 D}=n(0)$ for any $n_{s}$. Thus, we have to return to this problem again.

We are interested in the accumulation layer near an interface of STO. We then consider the case when the axis $x$ is directed perpendicular to the interface (plane $x=0$ ) and lies along the [100] axis of a cubic crystal of STO. Electrons 
are located near the surface due to the attractive potential of positive charges $e n_{s}$ near the interface. These charges create an external field $D_{0}=4 \pi e n_{s}$ (here and below, we use cgs units) applied from the outside of STO, which is directed along the $x$ axis. In that case, the problem is effectively onedimensional. If the electron three-dimensional density is denoted by $n(x)$, then the potential depth profile $\varphi(x)$ in the system is determined by the equations:

$$
\frac{d D}{d x}=-4 \pi e n(x), \quad D=E+4 \pi P, \quad \frac{d \varphi}{d x}=-E,
$$

where $D(x), E(x), P(x)$ are the electric displacement, the electric field, and the polarization in STO, respectively. Eq. (2) should be solved using proper boundary conditions. For an accumulation layer, the boundary conditions are $D(0)$ $=D_{0}$ and $\varphi(\infty)=0$.

To solve the system of Eq. (2), one also needs to know the two material relationships $E(P)$ and $\rho(\varphi)$. Let us start from the lattice dielectric response $E(P)$. STO is well known as a quantum paraelectric, where the onset of ferroelectric order is suppressed by quantum fluctuations. A powerful approach to describe the properties of ferroelectric-like materials is based on the Landau-Ginzburg theory. For a continuous second-order phase transition, the Landau-Ginzburg expression of the free energy density $F(x)$ is represented as a power series expansion with respect to the polarization $P$

$$
F(x)=F_{0}+\frac{2 \pi}{\kappa} P(x)^{2}+\frac{1}{4} A \frac{1}{P_{0}^{2}} P(x)^{4},
$$

where $F_{0}$ stands for the free energy density at $P=0, P_{0}$ $=e / a^{2}$ is the characteristic polarization, and the coefficient $\mathrm{A}$ describes the non-linear dielectric response. In general, $F$ depends on the components of the vector $P$, but in the chosen geometry, the problem is one-dimensional, and all vectors are directed along the $x$ axis. The crystal polarization $P$ is determined by minimizing the free energy density $F$ in the presence of the electric field $E$ where $\delta F / \delta P=E$. This condition relates $E$ and $P$. We note that $E \ll 4 \pi P$ and thus $D=E+4 \pi P$ $\simeq 4 \pi P$. Due to electric neutrality, the number of accumulated electrons has to compensate the external field $D_{0}$, i.e.,

$$
D_{0}=4 \pi e n_{s}=4 \pi e \int_{0}^{\infty} n(x) d x .
$$

To take into account the electron screening of the external field, we use the Thomas-Fermi approach in which the electron concentration $n(x)$ and the self-consistent potential profile $\varphi(x)$ are related as $e \varphi(x)+\varepsilon(x)=\varepsilon_{F}=0$, where

$$
\varepsilon(x)=\left(3 \pi^{2}\right)^{2 / 3} \frac{\hbar^{2}}{2 m^{\star}}[n(x)]^{2 / 3}
$$

is the chemical potential of the electron gas. (Here we consider lightly doped STO). Using Eqs. (3), (5), and (2), we arrive at:

$$
F(x)-F_{0}=\frac{3^{2 / 3} \pi^{4 / 3}}{5} \frac{\hbar^{2}}{m^{\star}} n(x)^{5 / 3}
$$

Using this relationship at the surface $x=0$ with $P(0) \equiv e n_{s}$ and $n(0) \equiv n_{3 D}$, we arrive at Eq. (1).

To conclude, we find that the dependence of the electron mobility, $\mu$, for LAO/STO-based interfaces on the threedimensional electron density near the interface, $n_{3 D}$, is akin to the electron mobility dependence on density for bulk doped STO. This observation implies that the same scattering mechanism dominates the charge transport in both systems. In particular, at low electron densities $n_{3 D}<N$ and the electron mobility is limited by background impurity concentrations in STO. On the other hand, in the bulk samples with $n_{3 D}>N$, the mobility collapses because scattering happens on intentionally introduced donors. For LAO/STO, the polar catastrophe which provides electrons is not supposed to provide random donors. The fact that nevertheless the mobility is the same says that for LAO/STO, the model of disorder free polar catastrophe should be revised.

The theoretical work was supported by the National Science Foundation through the University of Minnesota MRSEC under Award No. DMR-1420013. H. Tuller thanks the National Science Foundation for his support under Award No. DMR-1507047. K.V.R. was supported by the Russian Science Foundation under Grant No. 17-72-10072. The authors gratefully acknowledge discussions with $\mathrm{M}$. Bibes, C. Leighton, B. Jalan, H. Fu, and D. C. Vaz.

${ }^{1}$ J. Mannhart and D. G. Schlom, Science 327, 1607 (2010).

${ }^{2}$ D. G. Schlom and J. Mannhart, Nat. Mater. 10, 168 (2011).

${ }^{3}$ J. A. Sulpizio, S. Ilani, P. Irvin, and J. Levy, Annu. Rev. Mater. Res. 44, 117 (2014).

${ }^{4}$ M. Gabay and J.-M. Triscone, Nat. Phys. 9, 610 (2013).

${ }^{5}$ G. Cheng, M. Tomczyk, S. Lu, J. P. Veazey, M. Huang, P. Irvin, S. Ryu, H. Lee, C.-B. Eom, C. S. Hellberg, and J. Levy, Nature 521, 196 (2015).

${ }^{6}$ N. Reyren, S. Thiel, A. D. Caviglia, L. F. Kourkoutis, G. Hammerl, C. Richter, C. W. Schneider, T. Kopp, A.-S. Ruetschi, D. Jaccard, M. Gabay, D. A. Muller, J.-M. Triscone, and J. Mannhart, Science 317, 1196 (2007).

${ }^{7}$ A. D. Caviglia, S. Gariglio, N. Reyren, D. Jaccard, T. Schneider, M. Gabay, S. Thiel, G. Hammerl, J. Mannhart, and J.-M. Triscone, Nature 456, 624 (2008).

${ }^{8}$ G. E. D. K. Prawiroatmodjo, F. Trier, D. V. Christensen, Y. Chen, N. Pryds, and T. S. Jespersen, Phys. Rev. B 93, 184504 (2016).

${ }^{9}$ A. Brinkman, M. Huijben, M. van Zalk, J. Huijben, U. Zeitler, J. C. Maan, W. G. van der Wiel, G. Rijnders, D. H. A. Blank, and H. Hilgenkamp, Nat. Mater. 6, 493 (2007).

${ }^{10}$ S. Thiel, G. Hammerl, A. Schmehl, C. W. Schneider, and J. Mannhart, Science 313, 1942 (2006).

${ }^{11}$ D. V. Christensen, F. Trier, Y. Z. Chen, A. Smith, J. Nygard, and N. Pryds, Appl. Phys. Lett. 102, 021602 (2013).

${ }^{12}$ F. Trier, G. E. D. K. Prawiroatmodjo, Z. Zhong, D. V. Christensen, M. von Soosten, A. Bhowmik, J. M. G. Lastra, Y. Chen, T. S. Jespersen, and N. Pryds, Phys. Rev. Lett. 117, 096804 (2016).

${ }^{13}$ Y. Matsubara, K. S. Takahashi, M. S. Bahramy, Y. Kozuka, D. Maryenko, J. Falson, A. Tsukazaki, Y. Tokura, and M. Kawasaki, Nat. Commun. 7, 11631 (2016).

${ }^{14}$ Y. Z. Chen, N. Bovet, F. Trier, D. V. Christensen, F. M. Qu, N. H. Andersen, T. Kasama, W. Zhang, R. Giraud, J. Dufouleur, T. S. Jespersen, J. R. Sun, A. Smith, J. Nygrd, L. Lu, B. Bchner, B. G. Shen, S. Linderoth, and N. Pryds, Nat. Commun. 4, 1371 (2013).

${ }^{15}$ Y. Z. Chen, F. Trier, T. Kasama, D. V. Christensen, N. Bovet, Z. I. Balogh, H. Li, K. T. S. Thydn, W. Zhang, S. Yazdi, P. Norby, N. Pryds, and S. Linderoth, Nano Lett. 15, 1849 (2015).

${ }^{16}$ Y. Z. Chen, F. Trier, T. Wijnands, R. J. Green, N. Gauquelin, R. Egoavil, D. V. Christensen, G. Koster, M. Huijben, N. Bovet, S. Macke, F. He, R. Sutarto, N. H. Andersen, J. A. Sulpizio, M. Honig, G. E. D. K. Prawiroatmodjo, T. S. Jespersen, S. Linderoth, S. Ilani, J. Verbeeck, G. Van Tendeloo, G. Rijnders, G. A. Sawatzky, and N. Pryds, Nat. Mater. 14, 801 (2015). 
${ }^{17}$ Y. Kozuka, M. Kim, H. Ohta, Y. Hikita, C. Bell, and H. Y. Hwang, Appl. Phys. Lett. 97, 222115 (2010).

${ }^{18}$ M. Huijben, G. Koster, M. K. Kruize, S. Wenderich, J. Verbeeck, S. Bals, E. Slooten, B. Shi, H. J. A. Molegraaf, J. E. Kleibeuker, S. van Aert, J. B. Goedkoop, A. Brinkman, D. H. A. Blank, M. S. Golden, G. van Tendeloo, H. Hilgenkamp, and G. Rijnders, Adv. Funct. Mater. 23, 5240 (2013).

${ }^{19}$ Y. Xie, C. Bell, Y. Hikita, S. Harashima, and H. Y. Hwang, Adv. Mater. 25, 4735 (2013).

${ }^{20}$ A. Fête, C. Cancellieri, D. Li, D. Stornaiuolo, A. D. Caviglia, S. Gariglio, and J.-M. Triscone, Appl. Phys. Lett. 106, 051604 (2015).

${ }^{21}$ S. Zeng, W. Lu, Z. Huang, Z. Liu, K. Han, K. Gopinadhan, C. Li, R. Guo, W. Zhou, H. H. Ma, L. Jian, T. Venkatesan, and Ariando, ACS Nano 10, 4532 (2016).

${ }^{22}$ T. D. Sanders, M. T. Gray, F. J. Wong, and Y. Suzuki, Phys. Rev. B 91, 205112 (2015).

${ }^{23}$ Y. Chen, N. Pryds, J. E. Kleibeuker, G. Koster, J. Sun, E. Stamate, B. Shen, G. Rijnders, and S. Linderoth, Nano Lett. 11, 3774 (2011).

${ }^{24}$ C. S. Koonce, M. L. Cohen, J. F. Schooley, W. R. Hosler, and E. R. Pfeiffer, Phys. Rev. 163, 380 (1967).

${ }^{25}$ O. N. Tufte and P. W. Chapman, Phys. Rev. 155, 796 (1967).

${ }^{26}$ W. Gong, H. Yun, Y. B. Ning, J. E. Greedan, W. R. Datars, and C. V. Stager, J. Solid State Chem. 90, 320 (1991).

${ }^{27}$ A. Spinelli, M. A. Torija, C. Liu, C. Jan, and C. Leighton, Phys. Rev. B 81, 155110 (2010).

${ }^{28}$ X. Lin, C. W. Rischau, L. Buchauer, A. Jaoui, B. Fauque, and K. Behnia, npj Quantum Mater. 2, 41 (2017).

${ }^{29}$ A. Verma, A. P. Kajdos, T. A. Cain, S. Stemmer, and D. Jena, Phys. Rev. Lett. 112, 216601 (2014).
${ }^{30}$ M. Minohara, Y. Hikita, C. Bell, H. Inoue, M. Hosoda, H. K. Sato, H. Kumigashira, M. Oshima, E. Ikenaga, and H. Y. Hwang, pre-print arXiv:1403.5594 (2014).

${ }^{31}$ Y. Yamada, H. K. Sato, Y. Hikita, H. Y. Hwang, and Y. Kanemitsu, Appl. Phys. Lett. 104, 151907 (2014).

${ }^{32}$ A. Dubroka, M. Rössle, K. W. Kim, V. K. Malik, L. Schultz, S. Thiel, C. W. Schneider, J. Mannhart, G. Herranz, O. Copie, M. Bibes, A. Barthélémy, and C. Bernhard, Phys. Rev. Lett. 104, 156807 (2010).

${ }^{33}$ M. Stengel, Phys. Rev. Lett. 106, 136803 (2011).

${ }^{34}$ W-J. Son, E. Cho, B. Lee, J. Lee, and S. Han, Phys. Rev. B 79, 245411 (2009).

${ }^{35}$ G. Khalsa and A. H. MacDonald, Phys. Rev. B 86, 125121 (2012).

${ }^{36}$ S. Y. Park and A. J. Millis, Phys. Rev. B 87, 205145 (2013).

${ }^{37}$ K. V. Reich, M. Schecter, and B. I. Shklovskii, Phys. Rev. B 91, 115303 (2015).

${ }^{38}$ H. Fu, K. V. Reich, and B. I. Shklovskii, Phys. Rev. B 92, 035204 (2015).

${ }^{39}$ H. Fu, K. V. Reich, and B. I. Shklovskii, J. Exp. Theor. Phys. 122, 456 (2016).

${ }^{40}$ A. D. Caviglia, S. Gariglio, C. Cancellieri, B. Sacépé, A. Fête, N. Reyren, M. Gabay, A. F. Morpurgo, and J.-M. Triscone, Phys. Rev. Lett. 105, 236802 (2010).

${ }^{41}$ M. Ben Shalom, A. Ron, A. Palevski, and Y. Dagan, Phys. Rev. Lett. 105, 206401 (2010).

${ }^{42}$ W. Rice, P. Ambwani, M. Bombeck, J. D. Thompson, G. Haugstad, C. Leighton, and S. Crooker, Nat. Mater. 13, 481 (2014).

${ }^{43}$ C. Xu, C. Bumer, R. A. Heinen, S. Hoffmann-Eifert, F. Gunkel, and R. Dittmann, Sci. Rep. 6, 22410 (2016).

${ }^{44}$ P. Ambwani, P. Xu, G. Haugstad, J. S. Jeong, R. Deng, K. A. Mkhoyan, B. Jalan, and C. Leighton, J. Appl. Phys. 120, 055704 (2016).

${ }^{45}$ G. Herranz, F. Sanchez, N. Dix, M. Scigaj, and J. Fontcuberta, Sci. Rep. 2, 758 (2012). 